\title{
Classical and Quantum Structures of the Wave: Modelling the Controlled, Optimised, Continuum-System
}

Tafireyi Nemaura

Department of Clinical Pharmacology, University of Zimbabwe, Harare, Zimbabwe

Email: tnemaura@gmail.com

How to cite this paper: Nemaura, T. (2022) Classical and Quantum Structures of the Wave: Modelling the Controlled, Optimised, Continuum-System. Journal of Applied Mathematics and Physics, 10, 611-622. https://doi.org/10.4236/jamp.2022.103044

Received: February 7, 2022

Accepted: February 28, 2022

Published: March 3, 2022

Copyright $\odot 2022$ by author(s) and Scientific Research Publishing Inc. This work is licensed under the Creative Commons Attribution International License (CC BY 4.0).

http://creativecommons.org/licenses/by/4.0/ (c) (i) Open Access

\begin{abstract}
A projected plasma efavirenz concentration profile of a patient who had been on an orally administered $600 \mathrm{mg}$ daily dose of efavirenz is used as the variable that is a directing lead. A four compartmental model of ordinary differential equations is suggested and solved numerically. This model projects descriptors associated with concentration dispersion subject to trace and bounce constraints. The stimulant-action unit is responsible for directing the bounce. A description of the cross-sectional structure of the quantum is suggested. A dynamical system with an attractive (zero eigenvalue) subspace as a structural state is derived. The structure is an intensity-effect measure. The bounce's Jacobian Matrix has negative eigenvalues which correspond to $A$-actionstimulation, $S$-action-intensity and $P$-effect-stimulation and these leads govern the structure $(C)$. The three paths, action-stimulation, action-intensity and effect-stimulation lead to the structure of an effect-intensity.
\end{abstract}

\section{Keywords}

Bounce, Quantum, Control, Option, Continuum, Structure

\section{Introduction}

Bohr's principle of complementarity or the wave-particle duality is a realisation in quantum mechanics [1] [2]. Bohr believed in a physical entity with two faces that appear to us as a particle in some situations and a wave in others. On the other hand, De-Broglie believed that there is just one thing that is always a particle and a wave (at the same time) and in such a way that the properties of the particle observed are influenced by the wave structure of the system [3]. A proposed depositing bounce is defined and modelled [4]. The description of the 
depositing bounce is made. There is characterisation of the bounce as it moves along its trace components towards an open-field whose size time is the signal continuity or stability (loop $(A, S)$ ). The bounce signal forms a loop. Time $(t)$ can be considered as a characteristic closure or bind (value of the field or the field-cost) of the bounce and the trace as the expression of the bounce.

The bounce describes movement towards the open field, conventionally with the controlled-structure or the directed-structure $(A, C)$ moving across it and optimised-continuum or the co-directed-continuum $(S, P)$ moving along it. A state-space of the bounce or wave is defined for modelling the depositing bounce. It consists of two source states or the action states which are direction $(A)$ an apex and optimization objective $(S)$. Additionally, there are two effect states responsible for the continuum $(P)$ and the structure or network $(C)$ or the conic [4] [5] [6].

The boundary is the controlling and options action, which is a directed residuum. The energiser is the control, a bouncing unit. The environs are apparent signals and responsible for optimised-control of the bounce [4]. The option is given by the bounce length. The continuum or fabric is the environmental stimulant and the structure is the environmental intensity. The environment is defined as media a potential [4]. The media is a structured-continuum effect. The continuity or limit is the bounce continuum. The structure is a communications system of the bounce or the bounce network. The structure is a result of how a bounce changes as it moves along some "vector" in its input. It is a representation of possibly the rate of change when an energy-rich point in multi-dimensions moves in the direction of the open-field.

Control or thrust is the action stimulant the bounce-generator an action catalyst that is a variable. This is suggested to be a unitary action entity, which is a point trace of the bounce. Option or the cost function is an action intensity. An option is the bounce-synthesizer which is a linear trace of the bounce towards a field. The option/cost is potentially the reason why a bounce changes as it moves along some vector in its input. Space is a bounce basis-function which is a utility, a "value or benefit catalyst" of the bounce. The continuum plane is an environmental stimulant a bounce-locality or the bounce-territory/bounce-habitat. It is potentially a continuity effect plane, that is a planar trace of the bounce towards an open-field. The network is the bounce-structure and an environmental intensity. This is a structural trace of the bounce towards a field. This is the process or design function of the bounce.

In this work there is a suggestion that it is potentially beneficial to look at quantum as a cross-sectional structure of the bounce and is determined by the direction of the bounce (orientation). The bounce structure, is modelled from the three leads. The classical mechanics (related to the bounce structure) coincides with quantum mechanics (related to cross-sectional structure) when (directional) poles are across the open-field. The bounces considered in $\mathrm{Ne}$ maura [4] [5] [6] are conservative they move in a closed path, they are indepen- 
dent of bounce paths $(A+S+\bar{P}+\bar{C}=0)$. The bounce considered herein is nonconservative it depends on the link connection $(P)$ (that is $(A+S+\bar{P}+\bar{C}=\bar{P})$ ).

This work proposes a model of the bounce. It suggests the cross-sectional structure of the bounce as the quantum effect. It models bounce dimensions of four traces responsible for the control, synthesis, continuum and structure of the bounce. It proposes "space" as a synthesis operation and structure as a distributional operation.

\section{Methods}

Projected Pharmacokinetic (plasma concentration-time profile) in a patient on $600 \mathrm{mg}$ daily dose of efavirenz (a drug used in HIV-therapy) is used as an input (advective aggregate) to the depositing wave projection. The hourly projected plasma concentration is for a time period of $94 \mathrm{~h}$ and the projected $A U C_{\infty}=212 \mathrm{mg} \cdot \mathrm{h} \cdot 1^{-1} \quad$ [7]. The software used, is $\mathrm{R}$ and Mathematica.

There is dynamical system modelling of four bounce (wave) traces towards an open-field, the controller (valve) (the decision-maker) or directing-lead $(A)$ a polar-action (with two poles or agents the grantor $(\alpha=A)$ and receptor $\left.\left(\omega=A^{*}\right)\right)$ which is a variable and is function of time (size of stability of the signal (loop $A$, $S)$ ), (signal objective function-bounce utility) optimality-lead $(S)$ or a residuum action-connection an action-link, continuum-lead $(P)$ (locality) an effect-link and $(C)$ (structure-media objective) the process-function of the bounce the system-repose, order or network a polar-effect (sink).

\subsection{Bounce Model}

Let $B$ be the bounce with the following properties. The bounce is inferred to consist of the four paths, that define control, optimization, continuity and the network. There is consideration of these four paths towards an open-field with respect to size of flow $(t)$;

$$
\begin{gathered}
\text { Control }(B)=A, \\
\text { Utility }(B)=S, \\
\text { Locality }(B)=P, \\
\text { Structure }(B)=C .
\end{gathered}
$$

The mathematical equivalence of $A$ is a variable unit with two agents (grantor and attractor), $S$ is a utility function (basis-function) $(S: X \rightarrow \mathbb{R}, X=(A, P, C)$ consumption or integration set), $X$ is the bounce-value's domain. $S$ is the heterogeneous catalyst or the surface-catalyst. The bounce-territory is given by $P$ is a continuity derived property (bounce-locality), and $C$ the process function is the shape of a derived property (bounce-structure) $(C: Y \rightarrow \mathbb{R}, Y=(A, P, S)$ consumption or integration set), $Y$ is the bounce-value's domain. The structure is the media's value.

The entity $A$ is the control or stimulating ability of the point that is the sensi- 
tivity or stimulation generator or the bounce-energiser, $S$ is the option that is the intensity generator, $C$ is the structure or the networking property in the point that is a flux generation (a polar-connection), and $P$ is the continuity property in the point which is the continuum or the fabric generation (link-connection). The bounce traces are projected to act into an open-field represented in Figure 1. The leads $P$ and $S$ are links running as latitudes and $A$ and $C$ running longitudinally.

These four paths are inferred to move along the following paths, $A$-follows a boundary's longitude (with ordered poles across time), $S$-follows a boundary's latitude (time similitude), $P$-follows the media's latitude (time similitude), and $C$-follows the media's longitude (with ordered poles across time). The open-field is inferred to have potentially mainly three distinct movement categories, where $m$ is the horizontally directed bounce along (poles along) the open field, $i$ is the vertically directed bounce across (poles across) the open field, and $r$ is the tilted directed bounce (poles tilt) to the open-field. The profile for the horizontal crosssectional structure suggests the wave aspect. The slanted directed bounce suggests a particle and the wave at the same time. The particle aspect is inferred for the vertical bounce (Figure 2).

The bounce considered here has two important assumptions:

1) action-intensity is equal to effect-stimulation;

$$
S=P .
$$

2) The wave totality's displacement or the mechanical thrust of the bounce being equal to fabric displacement or effect-stimulation the non-conservative bounce depends on the path of the link-connection $(P)$ a spread medium;



Figure 1. A representation of the bounce's field (a) $A$-(unit) and $C$-(net) are instantaneous in the field; (b) $P$-(flow) and $S$-(option) are momentus in the field. 


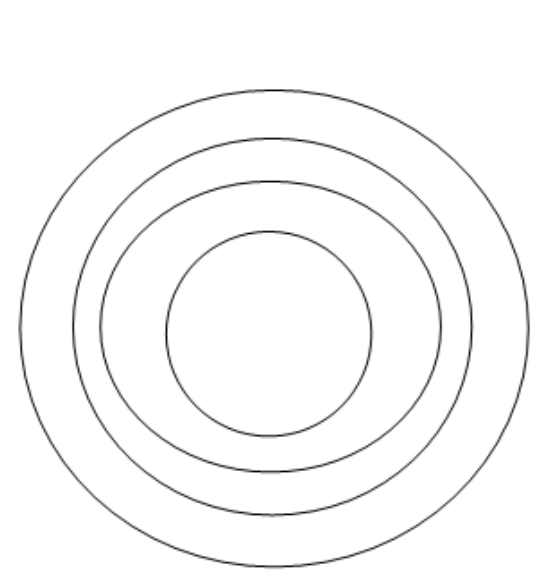

(a)

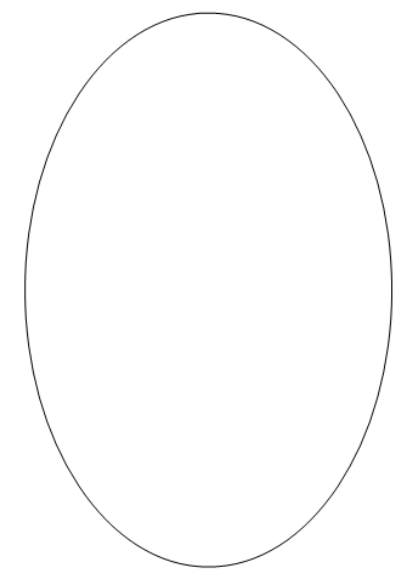

(c)

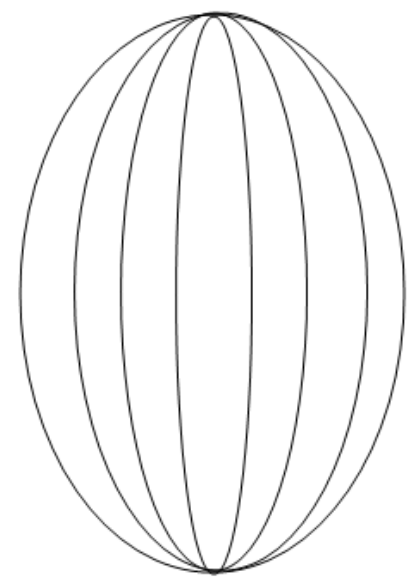

(b)

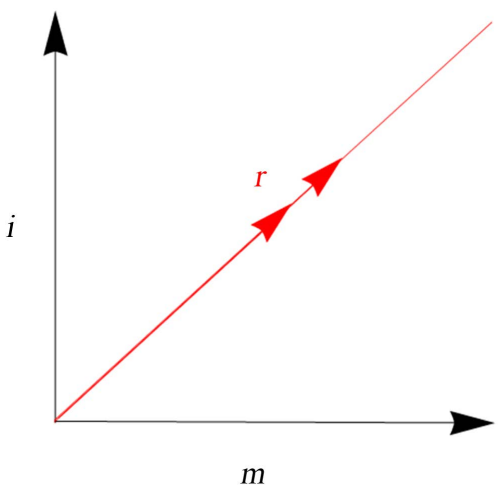

(d)

Figure 2. The simple cross-sectional structure of the bounce showing the projected structural "leaves" (electrons) and the directed bounces. (a) Horizontal cross-section structure (The spread- $h o_{m}$ ) along $m$ the horizontally directed bounce; (b) Sectional cross-section structure (The conic-spread- $s l_{r}$ ) along $r$ the oblique or slant directed bounce; (c) Vertical cross-section structure (The conic- $v e_{i}$ ) along $i$ the vertically directed bounce; (d) The orientated bounces.

$$
A+S+\bar{P}+\bar{C}=\bar{P}
$$

This reduces to a controlled-optimised system,

$$
A+S+\bar{C}=0 .
$$

In general, the depositing movement system (with the pole responsible for collection and the link responsible for spread) is such that:

$A$-Pole Boundary-Causal control environ or the bounce control;

$S$-Link Boundary-Causal utility environ or the bounce optimality;

$\bar{P}$-Link Medium-Effect flow or locality centre (effect-head) or the bounce continuity;

$\bar{C}$-Pole Medium-Effect structure centre (effect-tail) bounce order.

$$
\frac{\mathrm{d} A}{\mathrm{~d} t}=\gamma_{A \bar{C}}\left(A-A^{*}\right)-\gamma_{A S} A+\gamma_{S A}\left(\bar{S}+S_{\infty}\right),
$$




$$
\begin{gathered}
\frac{\mathrm{d} S}{\mathrm{~d} t}=\gamma_{A S} A-\gamma_{S A}\left(\bar{S}+S_{\infty}\right), \\
\frac{\mathrm{d} \bar{P}}{\mathrm{~d} t}=-\gamma_{A \bar{P}} A+\gamma_{\bar{P} A}\left(\bar{P}+P_{\infty}\right), \\
\frac{\mathrm{d} \bar{C}}{\mathrm{~d} t}=-\gamma_{A \bar{C}}\left(A-A^{*}\right) . \\
\gamma_{A \bar{C}}=k_{e}=\frac{C L}{V}=\frac{P_{\infty}}{A U C_{\infty} \cdot V}
\end{gathered}
$$

The parameters are defined as follows:

$\gamma_{A \bar{C}}$-depositing movement aggregate rate proportional constant.

$\gamma_{A \bar{P}}$-depositing movement part rate constant of $A$ into $\bar{P}$.

$\gamma_{\bar{P} A}$-response depositing movement part rate constant of $\bar{P}$ into $A$.

$A^{*}=0$ is the value of the pole receptor.

$P_{\infty}$-is the maximum stimulation-effect.

$S_{\infty}$-is the maximum intensity-action.

$A U C_{\infty}$-is the shape constant.

$C L$-Clearance parameter.

$V$-Unitary volume.

The reduced form (with $S=P$ ) is given by;

$$
\begin{gathered}
\frac{\mathrm{d} A}{\mathrm{~d} t}=\gamma_{A \bar{C}}\left(A-A^{*}\right)-\gamma_{A S} A+\gamma_{S A}\left(\bar{S}+S_{\infty}\right), \\
\frac{\mathrm{d} S}{\mathrm{~d} t}=\gamma_{A S} A-\gamma_{S A}\left(\bar{S}+S_{\infty}\right), \\
\frac{\mathrm{d} \bar{C}}{\mathrm{~d} t}=-\gamma_{A \bar{C}}\left(A-A^{*}\right) .
\end{gathered}
$$

The non-conservative bounce of the system is given by;

$$
\begin{gathered}
\frac{\mathrm{d} \dddot{A}}{\mathrm{~d} t}=\gamma_{A \bar{C}} \dddot{A}-\gamma_{A S} \dddot{A}+\gamma_{S A} \dddot{S}=\gamma_{A \bar{C}} \dddot{A}-\gamma_{A S} \dddot{A}-\gamma_{S A} \dddot{S}, \\
\frac{\mathrm{d} \dddot{S}}{\mathrm{~d} t}=\gamma_{A S} \dddot{A}-\gamma_{S A} \dddot{S}=\gamma_{A S} \dddot{A}+\gamma_{S A} \dddot{S}, \\
\frac{\mathrm{d} \dddot{\bar{C}}}{\mathrm{~d} t}=-\gamma_{A \bar{C}} \dddot{A} .
\end{gathered}
$$

\subsection{Equilibrium Points, Eigenvalues and Eigenvectors}

Setting,

$$
\frac{\mathrm{d} \dddot{A}}{\mathrm{~d} t}=\frac{\mathrm{d} \dddot{S}}{\mathrm{~d} t}=\frac{\mathrm{d} \dddot{\bar{C}}}{\mathrm{~d} t}=0
$$

the equilibrium points are given by,

$$
\dddot{A}^{*}=\dddot{S}^{*}=\dddot{\bar{C}}^{*}=0 .
$$

The Jacobian matrix for the field inter-system is given by, 


$$
\mathbb{J}\left(\dddot{A}^{*}, \dddot{S}^{*}, \dddot{C}^{*}\right)=\left[\begin{array}{ccc}
\gamma_{A \bar{C}}-\gamma_{A S} & -\gamma_{S A} & 0 \\
\gamma_{A S} & \gamma_{S A} & 0 \\
-\gamma_{A \bar{C}} & 0 & 0
\end{array}\right]_{\left(\dddot{A}^{*}, \dddot{S}^{*}, \dddot{C}^{*}\right)}
$$

The Jacobian determinant describes the volume dilation in the neighbourhood of a point. The volume dilation at equilibrium is such that,

$$
0=|\mathbb{J}-\lambda \mathbb{I}|,
$$

and is described by the characteristic equation of the field system given by,

$$
\lambda^{3}-\left(\gamma_{A \bar{C}}-\gamma_{A S}+\gamma_{S A}\right) \lambda^{2}+\gamma_{A \bar{C}} \gamma_{S A} \lambda=0 .
$$

The eigenvalues are given by:

$$
\begin{gathered}
\lambda_{1}=\frac{1}{2}\left(\gamma_{A \bar{C}}-\gamma_{A S}+\gamma_{S A}-\sqrt{\left(\gamma_{A \bar{C}}-\gamma_{A S}+\gamma_{S A}\right)^{2}-4 \gamma_{A \bar{C}} \gamma_{S A}}\right), \\
\lambda_{2}=\frac{1}{2}\left(\gamma_{A \bar{C}}-\gamma_{A S}+\gamma_{S A}+\sqrt{\left(\gamma_{A \bar{C}}-\gamma_{A S}+\gamma_{S A}\right)^{2}-4 \gamma_{A \bar{C}} \gamma_{S A}}\right), \\
\lambda_{3}=0 .
\end{gathered}
$$

The associated eigenvectors are given by:

$$
\begin{gathered}
e_{1}=\{u, u v, 1\}, \\
e_{2}=\left\{u^{+}, u^{+} v^{+}, 1\right\},
\end{gathered}
$$

and

$$
e_{3}=\{0,0,1\},
$$

where $u, u^{+}, v, v^{+}$are given by:

$$
\begin{gathered}
u=-\frac{1}{2 \gamma_{A \bar{C}}}\left(\gamma_{A \bar{C}}-\gamma_{A S}+\gamma_{S A}-\sqrt{\left(\gamma_{A \bar{C}}-\gamma_{A S}+\gamma_{S A}\right)^{2}-4 \gamma_{A \bar{C}} \gamma_{S A}}\right), \\
u^{+}=-\frac{1}{2 \gamma_{A \bar{C}}}\left(\gamma_{A \bar{C}}-\gamma_{A S}+\gamma_{S A}+\sqrt{\left(\gamma_{A \bar{C}}-\gamma_{A S}+\gamma_{S A}\right)^{2}-4 \gamma_{A \bar{C}} \gamma_{S A}}\right), \\
v=\frac{1}{\gamma_{S A}}\left(\gamma_{A \bar{C}}-\gamma_{A S}\right)-\frac{1}{2 \gamma_{S A}}\left(\gamma_{A \bar{C}}-\gamma_{A S}+\gamma_{S A}-\sqrt{\left(\gamma_{A \bar{C}}-\gamma_{A S}+\gamma_{S A}\right)^{2}-4 \gamma_{A \bar{C}} \gamma_{S A}}\right), \text { (32) } \\
v^{+}=\frac{1}{\gamma_{S A}}\left(\gamma_{A \bar{C}}-\gamma_{A S}\right)-\frac{1}{2 \gamma_{S A}}\left(\gamma_{A \bar{C}}-\gamma_{A S}+\gamma_{S A}+\sqrt{\left(\gamma_{A \bar{C}}-\gamma_{A S}+\gamma_{S A}\right)^{2}-4 \gamma_{A \bar{C}} \gamma_{S A}}\right) .
\end{gathered}
$$

\section{Results}

Depositing bounce: Parameter Estimation, Equilibrium Points, Eigenvalues and Eigenvectors, and Numerical solutions

The projected hourly concentration in a 94 hour time period is used. The concentration aggregate is the input variable to obtain the parameter estimates (Table 1) of Equations (8) - (11).

The numerical solutions of the depositing bounce's traces, that is for the control (Advection) path $(A)$, Optimality path $(S)$ a saturation amplitude an action 
Table 1. Parameter estimates in modelling transportation rates of the particle model.

\begin{tabular}{ccccc}
\hline Parameters & Estimate & Std Error & t value & $\operatorname{Pr}(>|\mathbf{t}|)$ \\
\hline$P_{\infty}$ & $91.67 \mathrm{mg}$ & Fix & - & - \\
$V$ & 11 & Fix & - & - \\
$A U C_{\infty}$ & $212 \mathrm{mg} \cdot \mathrm{l}^{-1} \cdot \mathrm{h}$ & $8.475 \times 10^{-7}$ & $250,101,593$ & $<2 \times 10^{-16}$ \\
$\gamma_{A S}$ & 0.7096 & $2.607 \times 10^{-9}$ & $272,207,949$ & $<2 \times 10^{-16}$ \\
$\gamma_{S A}$ & 0.03145 & $2.932 \times 10^{-10}$ & $107,256,860$ & $<2 \times 10^{-16}$ \\
\hline
\end{tabular}

link, Continuum $(P)$ an effect link the passive aggregate, and structural aggregate (C) a convective amplitude are shown (Figure 3 ).

The equilibrium points, eigenvalues and eigenvectors are:

$$
\begin{gathered}
\left(A^{*}, S^{*}, \bar{C}^{*}\right)=(0,0,0), \\
\left(\lambda_{1}, \lambda_{2}, \lambda_{3}\right)=(-0.16158,-0.08416,0),
\end{gathered}
$$

and

$$
\begin{gathered}
e_{1}=(0.21479,-0.78960,0.57480), \\
e_{2}=(-0.12397,0.76089,-0.63692), \\
e_{3}=(0,0,1) .
\end{gathered}
$$

\section{Discussion}

A wave or bounce is proposed to be a directed residuum boundary with structured continuum media. The bounce considered herein is non-conservative. The bounce follows the continuum path and is such that optimality $(S)$ is equal to effect-supply or continuum $(P)$. The bounce is comprised of the bounce-control (a sensitivity-point, primary control), bounce-optimality (primary objective), bouncecontinuity (secondary control) and bounce-order (secondary objective) a distribution form or the shape. A wave can be described as a linking-function of the signal and the media in a field. Gravity (a secondary control) controls the media and the structure is a media objective a secondary objective. The structure is the media's value.

The direction or control of the bounce is a "point". It is considered as the stimulating-action $(A)$ a unitary-lead of the bounce or a signal-control. The utility of the bounce is potentially a linear aggregate an optimization-lead. This is an intensity-action $(S)$ lead and is the performance or "stability" measure of the bounce. The function $S$ is thus the bounce-catalyst the objective function of the bounce. It is important to note that space represents the value-benefit of the bounce. The environment is potentially suggested to be consisting of the flow $(P)$ and the net $(C)$. The continuum $(P)$ (gravity) is the flow-continuum (fabric locality) an expanse or the "media-control". This is a stimulating-effect lead. Finally, the structure $(C)$ is a network-repose of the bounce. The structure is an 


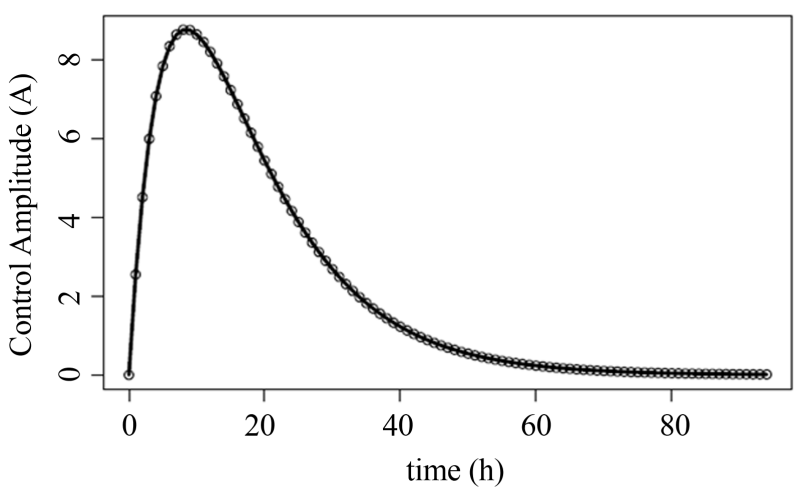

(a)

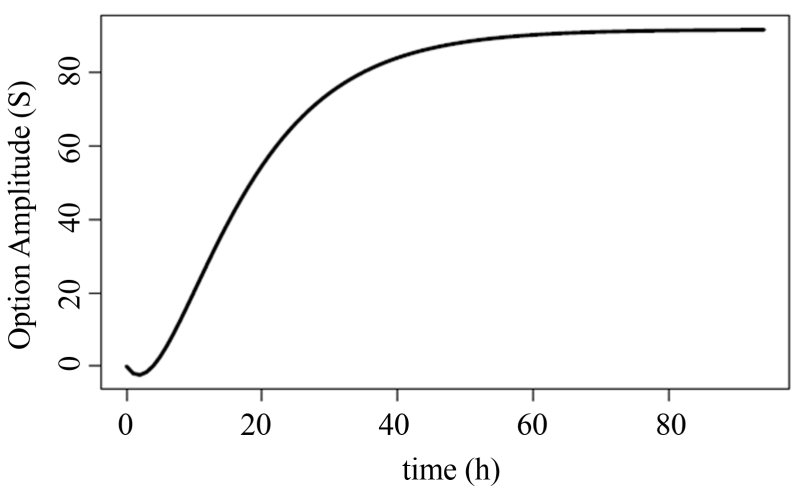

(c)

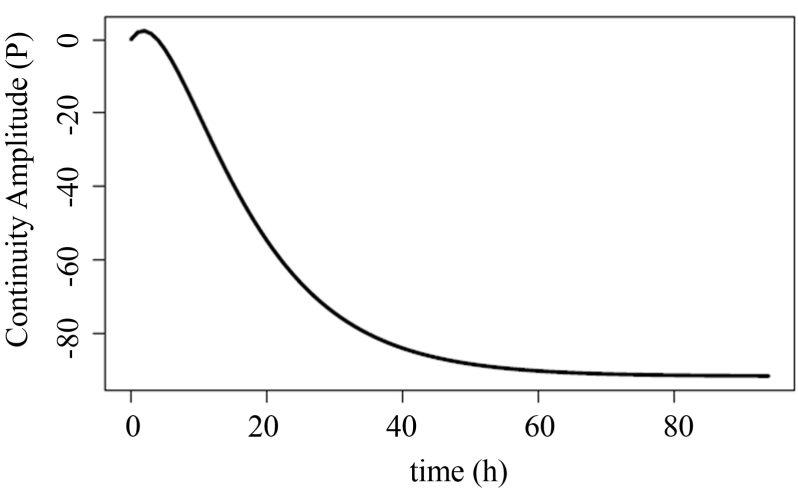

(b)

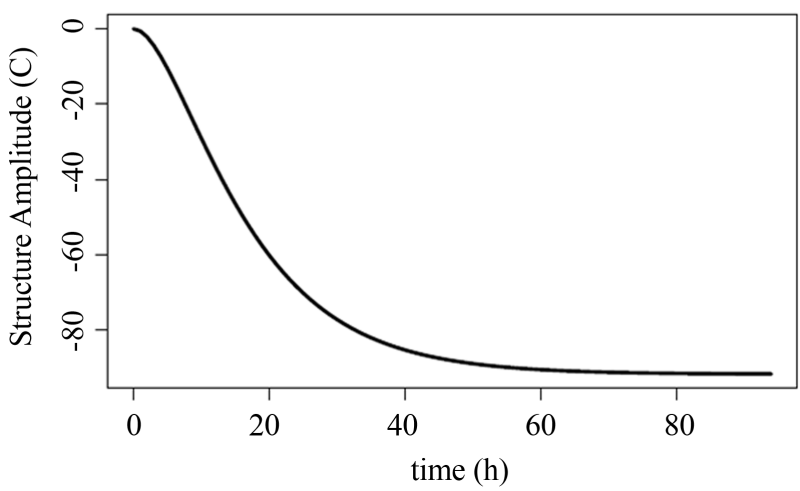

(d)

Figure 3. A representation of the bounce paths (a) $A$-the control path; (b) $\bar{P}$ : the continuum path; (c) $S$ : the options path; (d) $\bar{C}$ : the distribution path.

intensity-effect a repose of the bounce "media-cost" or the media's objective. The primary expressions are given by the boundary and secondary expressions are replicated by the media.

The wave is primarily controlled by its unit. In engineering and science, the control path can be inferred to be analogous to a neutron, an entity which is a controlling-action path. The neutron can be considered as a host and the constitutional-strain-environ. Furthermore, the neutron can be suggested to be a directing-environ $(A)$. On the other hand, a neutrino is possibly a linear-option that is a potential optimisation-action path. The neutrino is inferred to be an options-environ $(S)$. A proton can be suggested to be an expanse-plane or a continuous-effect path marking the bounce-flow. The proton is possibly a medium and a supply-stress-environment $(P)$. Furthermore, an electron is inferred to be a structure or a gathered-effect path. The electron is potentially a medium and the distribution-stress-environment. The electron is potentially a structural/network center or dispersion-centre $(C)$. The structural "leaves" are inferred to be the horizontal form of electrons in the bounce identified as "wave form" [4]. The surface is taken to be an exterior of the bounce given by $A, S$ and $P$ and the interface is a core a "tension drop" given by $C$.

The depositing bounce considered herein had two invariant "spaces", a fast 
subspace (a bounce-surface) with negative eigenvalues corresponding to controllability, optimality, and continuity and a slow subspace an interface (a bounceinterface) corresponding to the zero eigenvalue an attractive path of equilibra coresponding to the structure or the network (A dispersal path that forms a sigmoidal relationship is derived. The fast subspace forms a rigid surface (exterior-(surface and medium)) and the structure is a yielding interface (interiormedium) (effect-in-interface).

The hypothesis suggested by Bohr caters for the vertical and horizontal bounce and De-Broglie is supported by the slanted bounce, which is a resultant bounce to Bohr's assertions. This work however attempts to clarify the circumstances as suggested by Bohr leading to changes in observations. While De-Broglie suggests the wave structure as a guide. In this work, there are suggestions that the changes observed are due to the direction of the poles (pole-orientation) brought on by the signal catalyst $(S)$ and these are significant in determining the cross-sectional structure of the bounce. Additionally, the orientation (horizontal, vertical, oblique) of the wave structure (bounce) can be used as a reference in informing the cross-sectional structure. The cross-sectional structure is the observed aspect in "quantum" mechanics [3]. In quantum, the bounce structure is an independent effect and what changes are the direction of the bounce (bounce orientation). Thus, altering the quantum.

What then is quantum? It is the cross-sectional structure of the bounce's structure. Quantum is thus a function of direction or orientation of the unit. A domain for the direction $A$, can potentially be adopted $\operatorname{Dom}(A)=\{r, i, m\}$ and the range of quantum could be given by the structural cross-section, a co-domain $R_{A}=\left\{h o_{m}, v e_{i}, s l_{r}\right\}$. The De-Broglie assertions are potentially supported by $r$ and the Bohr's assertions are supported by $i$ and $m$. Quantum mechanics is the mechanics of the cross-section of the bounce structure. The mechanics of the bounce structure the macro-state is different from the mechanics of its cross-section a micro-state. The classical mechanics aligns to quantum mechanics when poles are across in the $i$ bounce orientation. This can be referred to as similar to the conventional mode. Polar $(A, C)$ bounce movements are instantaneous and link $(S, P)$ bounce movements are momentus.

An analogous model to the bounce structure is given by the onion and its cross-sections, the onion rings [8] [9]. Another important analogy of the bounce is given by lattices, where the continuum $(P)$ can be considered as an absorption law on a set of two elements (grantors $(A)$ and receptors $\left(A^{*}\right)$ ) with the two operations which are synthesis or combinations $(S)$ and distribution $(C)$. The bounce thus forms a lattice [10].

\section{Conclusion}

A four dimensional model can be used to understand the signal and media movement in a bounce. A cross-sectional structure of the profile of the modelled bounce is suggested. It is suggested to be the quantum structure. The modelling 
considered herein shows how to incorporate and define constraints in modelling the bounce. It further suggests that the wave can be considered to have two main objectives related to the signal component which is "space" and the other to the medium which is the structure. A wave can thus be modelled by a bi-criteria optimisation function. The two are objective functions and connected with time. The wave has thus boundary and media parts [4]. The boundary has a polychromatric control of the signal and an objective option. The media has a monochromatic control of the "state" and forms an objective structure. A wave or bounce is proposed to be a directed residuum boundary with structured continuum media. A non-conservative bounce is used to model the three leads that govern the structure. A wave has provisionally two outputs space and structure. "Space" is a catalyst and explains why the bounce changes and the structure is the procedural output of the bounce. There is a conclusion that signal value is the catalyst of the basis and structure (media's value) is the product of the process. Time binds these two "value" functions (basis and process functions).

\section{Acknowledgements}

The author would like to thank the following; C. Nhachi, C. Masimirembwa, and G. Kadzirange, AIBST and The College of Health Sciences, University of Zimbabwe.

\section{Conflicts of Interest}

The author declares no conflicts of interest regarding the publication of this paper.

\section{References}

[1] Bohr, N. (1928) The Quantum Postulate the Recent Development of Atomic Theory. Nature, 121, 580-590. https://doi.org/10.1038/121580a0

[2] Lü, X. (2020) Quantitative Wave-Particle Duality as Quantum State Discrimination. Physical Review A, 102, Article No. 022201. https://doi.org/10.1103/PhysRevA.102.022201

[3] Diner, S., Fargue, D., Lochak, G. and Selleri, F. (2012) The Wave-Particle Dualism: A Tribute to Louis de Broglie on His 90th Birthday (Vol. 3). Springer Science \& Business Media.

[4] Nemaura, T. (2020) A Theoretical Model of a Wave: An Efflux Bounce of a SolutionParticle. Journal of Physics Communications, 4, Article ID: 105009. https://doi.org/10.1088/2399-6528/abc056

[5] Nemaura, T. (2018) Derived Wave of Gradient Driven Diffusion's Convective Flux of Efavirenz. Journal of Physics Communications, 2, Article ID: 055008. https://doi.org/10.1088/2399-6528/aac0f0

[6] Nemaura, T. (2019) Modelling the Influx and Efflux Waves in Drug Movement: A Basis for Pharmacokinetic-Pharmacodynamic Link of Efavirenz. Biomedical Physics \& Engineering Express, 6, Article ID: 015002. https://doi.org/10.1088/2057-1976/ab559b

[7] Urso, R., Blardi, P. and Giorgi, G. (2002) A Short Introduction to Pharmacokinetics. 
European Review for Medical and Pharmacological Sciences, 6, 33-34.

[8] Krishnan, K.N. (2002) On the Formation of Onion Rings in Friction Stir Welds. Materials Science and Engineering: A, 327, 246-251. https://doi.org/10.1016/S0921-5093(01)01474-5

[9] Niedderer, H. and Petri, J. (1995) Student Learning Processes in a Computer-based Teaching Approach of Quantum Physics. In: Hofstein, A., Ed., Science Education: From Theory to Practice, Weizmann Institute of Science, Rehovot, Israel, 145 p.

[10] Liu, Y.E. (2020) Discussion on Propositional Logic Incorporating Set Thought into Discrete Mathematics. Journal of Physics. Conference Series, 1634, Article No. 012087. https://doi.org/10.1088/1742-6596/1634/1/012087 